\title{
NUMERICAL SOLUTION FOR MAGNETOHYDRODYNAMIC STAGNATION POINT FLOW TOWARDS A STRETCHING OR SHRINKING SURFACE IN A SATURATED POROUS MEDIUM
}

\author{
Susheela Chaudhary ${ }^{1}$, Sawai Singh ${ }^{2}$, Santosh Chaudhary ${ }^{3}$ \\ ${ }^{1,2}$ Department of Mathematics \\ S.K. Govt. (P.G.) College \\ Sikar, 332001, INDIA \\ ${ }^{3}$ Department of Mathematics \\ Malaviya National Institute of Technology \\ Jaipur, 302017, INDIA
}

\begin{abstract}
Mathematical analysis is carried out to investigate two-dimensional flow of a viscous incompressible electrically conducting fluid near a stagnation point of a stretching or shrinking surface in a saturated porous medium. Defining equations are formulated to determine the scope of considered variables and governing partial differential equations are reduced to ordinary differential equations by using suitable similarity transformation. $\mathrm{Nu}-$ merical computation of the problem was performed by shooting iteration technique together with Runge-Kutta fourth order integration scheme. Effects of Prandtl number, permeability parameter, magnetic parameter and stretching or shrinking parameter on velocity and temperature profiles are computed and illustrated graphically, whereas numerical values of local skin-friction coefficient and local Nusselt number are presented in tabular form.
\end{abstract}

AMS Subject Classification: 76SXX, 76S05

Key Words: magnetohydrodynamic flow, stagnation point, stretching surface, shrinking surface, porous medium

Received: September 27, 2015

Published: March 13, 2016

$\S_{\text {Correspondence author }}$ (c) 2016 Academic Publications, Ltd. url: www.acadpubl.eu 


\section{Introduction}

Flow and heat transfer of viscous incompressible fluid over stretching sheet has extensive theoretical and technical applications in manufacturing processes in industries such as glass fiber production, artificial fibers, manufacturing plastic films, aerodynamic extrusion of plastic sheets, extrusion of polymer in a meltspinning process, crystal growing, cooling and drying of paper and textiles etc. This study is gaining interest due to its applications in designing of cooling systems such as flow meters, pumps, liquid metals and MHD generators. Many chemical engineering processes, such as metallurgical and polymer extrusion, involve cooling of molten liquids being stretched into cooling liquids. [1] was the first to consider steady two-dimensional boundary layer flow of viscous incompressible fluid past a stretching plate and obtained an exact similarity solution in closed analytical form. Effects of heat transfer on a stretching surface under different aspects of the problem have been investigated by many authors such as [2]-[7].

The study of stagnation point flow is of extensive technical importance in the field of drag reduction, thermal oil recovery, design of thrust bearings and radial diffusers, and transpiration cooling etc., due to the fact that science and technology continue to demand improved accuracy in practical applications. Besides, stagnation point flow has widely relevance in the field of high-performance applications of solar central receivers exposed to wind currents, cooling of nuclear reactors during emergency shutdown, cooling of electronic devices by fans, and many other hydrodynamic processes. Many authors in various studies have focused on stagnation point flow and described its subsequent uses in industrial and engineering applications such as continuous casting, insulating materials, extrusion, and glass drawing etc. Significant studies carried out by several researchers related to stagnation point flow over stretching surface taking into account various physical features and have been discussed in publications by [8]-[13].

Moreover, the shrinking sheet effect on the flow of an incompressible fluid has attracted attention of researchers due to the fact that the flow induced by shrinking sheet exhibits significantly different physical phenomena from the forward stretching flow in the boundary layer. This effect is found relevant in many practical applications of textile industries, paper production, polymer processing, manufacturing of glass sheets etc. Shrinking film is one of the common applications of shrinking problems encountered in engineering, industries, agricultural development and environmental strategies. In packaging of bulk products it is useful because of its property that it can be unwrapped eas- 
ily with the adequate heat. The study of the capillary effects in small pores, shrinking-swell behaviour, hydraulic and mechanical properties of agricultural clay soils involves influence of shrinking film phenomena in prediction of flow and transport processes through fine grained soils. In addition, a rising balloon also subjected to the effect of the shrinking surface phenomenon. [14] studied stagnation flow towards a shrinking sheet and investigated solutions of the problem that do not exist for large shrinking rates. Recently, [15]-[17] described the problem of flow past a shrinking surface in various ways.

Flow past a surface embedded in a porous medium has potential practical consequences in many new areas of engineering and geophysics such as insulation of buildings, geothermal energy utilization, energy storage and many others. Some other quite promising applications are in the field of metallurgy like magnetic-levitation casting and MHD stirring of molten metal. Besides, it has growing scope in the field of optimization of the solidification processes of metals and metal alloys, study of geothermal source, control of underground spreading of chemical wastes and pollutants, design of MHD power generators, design of heat exchangers and MHD accelerators etc. Further, mixed convection flow of an electrically conducting fluid over a porous saturated flat surface in the presence of a magnetic field is also of special technical significance due to its frequent occurrence in many industrial applications such as microelectronic devices, MHD marine propulsion and electronic packages etc. The study in these context and its implications has been reviewed in the books by [18]-[21]. Several researchers such as [22]-[27] considered the flow problem in porous media under various situations and obtained similarity solutions. Recently, [28] studied magnetohydrodynamic stagnation point flow past a porous stretching surface with heat generation and described the effect of various parameters.

In view of the substantial investigations mentioned above and looking into importance of increasing practical applications of MHD effects in engineering and technology, the main objective of present study is to extend the problem of [25] for an electrically conducting fluid in the presence of a uniform transverse magnetic field with defined boundary conditions.

\section{Model Formulation}

Consider a steady two-dimensional boundary layer flow $(u, v, 0)$ of a viscous incompressible electrically conducting fluid near a stagnation point over a stretching or shrinking surface placed in the plane $y=0$ of a Cartesian coordinate system in a saturated porous medium. The $x$ - axis is taken along the surface 
with origin at the stagnation point and $y$ - axis is perpendicular to it. The flow is assumed to be confined in half plane $y>0$. It is also assumed that the surface is stretched or shrunk in its own plane with velocity proportional to the distance from the stagnation point in the presence of an externally applied normal magnetic field of constant $\operatorname{strength}\left(0, B_{0}, 0\right)$ as shown in Figure 1 (a) and Figure 1 (b). The magnetic Reynolds number is taken to be very small such that the induced magnetic field is negligible. The stretching or shrinking surface has velocity $u_{w}(x)=b x$, and surface temperature $T_{w}$, while the external flow has velocity $u_{e}(x)=a x$ and temperature $T_{\infty}$, where $x$ is the coordinate measured along the surface, $b$ is the stretching or shrinking rate, with $b>0$ for stretching case and $b<0$ for shrinking case and $a>0$ is the strength of the stagnation flow. The system of boundary layer equations in a porous medium is given by:

$$
\begin{aligned}
& \frac{\partial u}{\partial x}+\frac{\partial v}{\partial y}=0 \\
& u \frac{\partial u}{\partial x}+v \frac{\partial u}{\partial y}=u_{e} \frac{d u_{e}}{d x}+v \frac{\partial^{2} u}{\partial y^{2}}+\frac{v}{K_{1}}\left(u_{e}-u\right)+\frac{\sigma_{e} B_{0}^{2}}{\rho}\left(u_{e}-u\right), \\
& u \frac{\partial T}{\partial x}+v \frac{\partial T}{\partial y}=\alpha \frac{\partial^{2} T}{\partial y^{2}}
\end{aligned}
$$

with the boundary conditions:

$$
\begin{aligned}
& y=0: u=u_{w}(x)=b x, v=0 ; \\
& T=T_{w} \text { and } y \rightarrow \infty: u=u_{e}(x)=a x ; \\
& T=T_{\infty} .
\end{aligned}
$$

where $y$ is the coordinate measured along perpendicular to the surface, $v=\frac{\mu}{\rho}$ is the kinematic viscosity, $\mu$ is the coefficient of fluid viscosity, $\rho$ is the fluid density, $K_{1}$ is permeability of the porous medium, $\sigma_{e}$ is the electrical conductivity, $T$ is the temperature of the fluid and $\alpha$ is thermal diffusivity.

\section{Similarity Transformation}

The continuity equation (1) is identically satisfied by introducing a stream function $\psi(x, y)$ such that

$$
u=\frac{\partial \psi}{\partial y}, \quad v=-\frac{\partial \psi}{\partial x}
$$




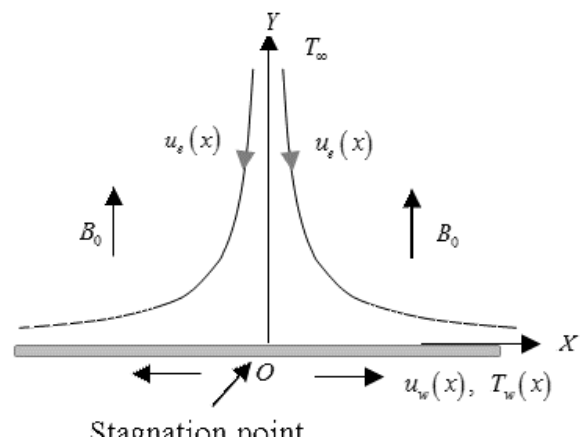

(a)

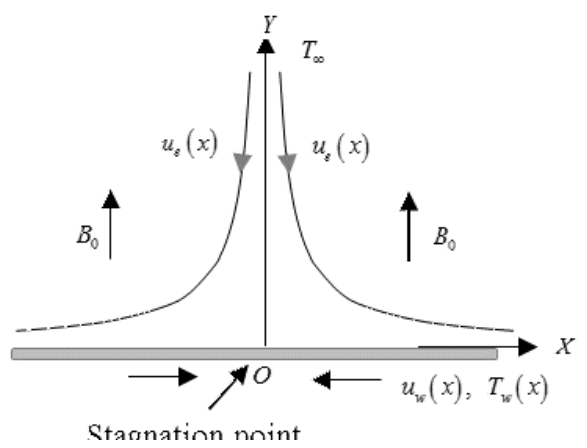

(b)

Figure 1: (a) Physical modal of two-dimensional stagnation point flow over stretching surface, (b) Physical modal of two-dimensional stagnation point flow over shrinking surface.

The momentum and energy equations (2) can be transformed into the corresponding ordinary differential equations by introducing the following dimensionless variables [25] as:

$$
\psi(x, y)=\sqrt{a \alpha} x f(\eta), \quad \eta=\sqrt{\frac{a}{\alpha}} y ; \theta(\eta)=\frac{T-T_{\infty}}{T_{w}-T_{\infty}},
$$

where $f(\eta)$ is the dimensionless stream function, $\eta$ is the similarity variable and $\theta(\eta)$ is the dimensionless temperature.

Substituting equations (4) and (5) in equations (2), the governing equations reduce to:

$$
\operatorname{Pr} f^{\prime \prime \prime}+f f^{\prime \prime}-f^{\prime 2}+K\left(1-f^{\prime}\right)+M\left(1-f^{\prime}\right)+1=0 \text { and } \theta^{\prime \prime}+f \theta^{\prime}=0,
$$

with the boundary conditions:

$$
\eta=0: f=0, f^{\prime}=\frac{b}{a}=c ; \theta=1 \text { and } \eta \rightarrow \infty: f^{\prime} \rightarrow 1 ; \theta \rightarrow 0,
$$

where primes denote differentiation with respect to $\eta, \operatorname{Pr}=\frac{v}{\alpha}$ is the Prandtl number, $K=\frac{v}{a K_{1}}$ is the permeability parameter, $M=\frac{\sigma_{e} B_{0}^{2}}{\rho a}$ is the magnetic parameter ad $c=\frac{b}{a}$ is the stretching parameter when $c>0$ or shrinking parameter when $c<0$, while $c=0$ is the planar stagnation flow towards a stationary surface. Moreover, $c=1\left(u_{w}=u_{e}\right)$ corresponds to the flow with no boundary layer. 


\section{Numerical Scheme}

Equations (6) with boundary conditions (7) are solved using shooting method, by converting them to a system of initial value problem. The unknown initial conditions are guessed. These unknown initial conditions are then updated using the known boundary conditions at $\eta \rightarrow \infty$. In this technique, the unknown initial conditions at $\eta=0$ are estimated using an optimization procedure [White and Subramanian (29)]. We first convert equations (6) into following system of simultaneous equations of first order for five unknowns. We set

$$
\begin{aligned}
& f_{1}^{\prime}=f_{2}, \quad f_{2}^{\prime}=f_{3}, \\
& f_{3}^{\prime}=-\frac{1}{\operatorname{Pr}}\left\{f_{1} f_{3}-f_{2}^{2}+K\left(1-f_{2}\right)+M\left(1-f_{2}\right)+1\right\}, \\
& f_{4}^{\prime}=f_{5}, \quad f_{5}^{\prime}=-f_{1} f_{5},
\end{aligned}
$$

where $f_{1}=f, f_{2}=f^{\prime}, f_{3}=f^{\prime \prime}, f_{4}=\theta, f_{5}=\theta^{\prime}$, with the initial conditions:

$$
f_{1}(0)=0, \quad f_{2}(0)=c, \quad f_{3}(0)=m, \quad f_{4}(0)=1, \quad f_{5}(0)=n,
$$

where $m$ and $n$ are priori unknown to be determined as a part of the numerical solution.

In order to solve equations (8) as an initial value problem, we require five initial conditions. Here three initial conditions $f_{1}(0), f_{2}(0)$ and $f_{4}(0)$ are known while two initial conditions $f_{3}(0)$ i.e. $f^{\prime \prime}(0)$ and $f_{5}(0)$ i.e. $\theta^{\prime}(0)$ are unknown but no such values are given at the boundary. The values of $f^{\prime}(\eta)$ and $\theta(\eta)$ are known at $\eta \rightarrow \infty$. These two conditions are utilized to produce two unknown initial conditions at $\eta=0$ by using shooting technique. The suitable estimated values for $f^{\prime \prime}(0)$ and $\theta^{\prime}(0)$ are chosen and then integration is carried out by Runge-Kutta fourth order integration scheme. Comparing the calculated values for $f^{\prime}(\eta)$ and $\theta(\eta)$ at appropriate finite large value of $\eta=\eta_{\infty}$ (say), which depend on the values of the parameters considered, with the given boundary conditions $f^{\prime}\left(\eta_{\infty}\right)=1$ and $\theta\left(\eta_{\infty}\right)=0$ and adjust the estimated values of $f^{\prime \prime}(0)$ and $\theta^{\prime}(0)$, to get a better approximation for the solution. After knowing all the finite initial conditions, we solve equations (8) by using shooting iteration technique together with Runge-Kutta fourth order integration scheme with step-size 0.001. The above procedure is repeated until we get the converged results up to the desired degree of accuracy $10^{-5}$. The numerical values of velocity and temperature profiles are plotted in Figures 2 to 5 and 6 to 9 respectively. 


\section{Skin-Friction Coefficient and Surface Heat Flux}

The physical quantities of interest are the local skin-friction coefficient $C_{f}$ and local Nusselt number $N u_{x}$, which are defined as:

$$
C_{f}=\frac{\tau_{w}}{\rho u_{e}^{2} / 2}=\frac{\mu\left(\frac{\partial u}{\partial y}\right)_{y=0}}{\rho u_{e}^{2} / 2} ; \quad N u_{x}=\frac{x q_{w}}{\kappa\left(T_{w}-T_{\infty}\right)}=-\frac{x\left(\frac{\partial T}{\partial y}\right)_{y=0}}{T_{w}-T_{\infty}} .
$$

Which in the present case, can be expressed in the following forms

$$
C_{f}=2 \sqrt{\frac{\operatorname{Pr}}{R e_{x}}} f^{\prime \prime}(0) ; \quad N u_{x}=-\sqrt{P e_{x}} \theta^{\prime}(0),
$$

where $\tau_{w}=\mu\left(\frac{\partial u}{\partial y}\right)_{y=0}$ is wall shear stress, $q_{w}=-\kappa\left(\frac{\partial T}{\partial y}\right)_{y=0}$ is the surface heat flux, $\kappa$ is the thermal conductivity, $R e_{x}=\frac{u_{e} x}{v}$ is the local Reynolds number and $P e_{x}=\frac{u_{e} x}{\alpha}$ is the local Péclet number.

Numerical values of the wall shear stress $f^{\prime \prime}(0)$ and the heat transfer rate $\theta^{\prime}(0)$ at the surface for various values of the physical parameters are presented in Table 1.

\section{Results and Discussion}

The influence of variation of velocity profiles $f^{\prime}(\eta)$ against $\eta$ for various values of the Prandtl number Pr, the permeability parameter $K$, the magnetic parameter $M$ and the stretching or shrinking parameter $c$ are illustrated in Figures 2 to 5 respectively, while the other parameters are constant. These Figures reveal that velocity of the fluid decreases with increasing values of the Prandtl number $\mathrm{Pr}$, whereas reverse behaviour observed for increasing values of the permeability parameter $K$, the magnetic parameter $M$ and the stretching or shrinking parameter $c$.

Figures 6 to 9 show the variation of temperature profiles $\theta(\eta)$ against $\eta$ for various values of the Prandtl number $\operatorname{Pr}$, the permeability parameter $K$, the magnetic parameter $M$ and the stretching or shrinking parameter $c$ respectively, while the other parameters are constant. From these Figures, it is evident that temperature increases with increasing values of the Prandtl number Pr, whereas reverse phenomenon take place for increasing values of the permeability parameter $K$, the magnetic parameter $M$ and the stretching or shrinking parameter $c$. 
The numerical results of the local skin-friction coefficient $f^{\prime \prime}(0)$ and the local Nusselt number $-\theta^{\prime}(0)$ are presented by Table 1 for various values of the Prandtl number $\operatorname{Pr}$, the permeability parameter $K$, the magnetic parameter $M$ and the stretching or shrinking parameter $c$. It is observed that local skinfriction coefficient $f^{\prime \prime}(0)$ decreases with increasing values of the Prandtl number $\mathrm{Pr}$ and the stretching or shrinking parameter $c$, while the reverse phenomenon occurs for the permeability parameter $K$ and the magnetic parameter $M$. Further, it is evident that local Nusselt number $-\theta^{\prime}(0)$ is decreases with increasing values of the Prandtl number $\operatorname{Pr}$ and the magnetic parameter $M$, while reverse behaviour observed for the permeability parameter $K$ and the stretching or shrinking parameterc. Furthermore, it is noted that there is no appreciable effect of increasing values of the permeability parameter $K$ on surface heat flux $\theta^{\prime}(0)$. In addition to, it is seen that the values of surface heat flux $\theta^{\prime}(0)$ is negative for all the values of physical parameters considered. Physically, negative value of surface heat flux $\theta^{\prime}(0)$ implies that there is a heat transfer from the surface.

\section{Conclusions}

Numerical analysis is carried out to study the problem of stagnation point flow of electrically conducting viscous incompressible fluid over a stretching or shrinking surface in a saturated porous medium. Governing partial differential equations for the flow and temperature fields are reduced to ordinary differential equations by using appropriate similarity transformation. Numerical computation of the problem was carried out by shooting iteration technique together with Runge-Kutta fourth order integration scheme. The following observations are found noteworthy in present study:

1. Velocity boundary layer thickness decreases with increasing values of the Prandtl number, while it increases with increasing values of the permeability parameter, the magnetic parameter and the stretching or shrinking parameter.

2. Thermal boundary layer thickness increases with increasing values of the Prandtl number, while it decreases with increasing values of the permeability parameter, the magnetic parameter and the stretching or shrinking parameter.

3. Wall shear stress decreases with increasing values of the Prandtl number and the stretching or shrinking parameter, whereas reverse behaviour 
observed for the permeability parameter and the magnetic parameter.

4. Heat transfer rate increases with increasing values of the Prandtl number and the magnetic parameter, while reverse phenomenon occurs for the permeability parameter and the stretching or shrinking parameter.

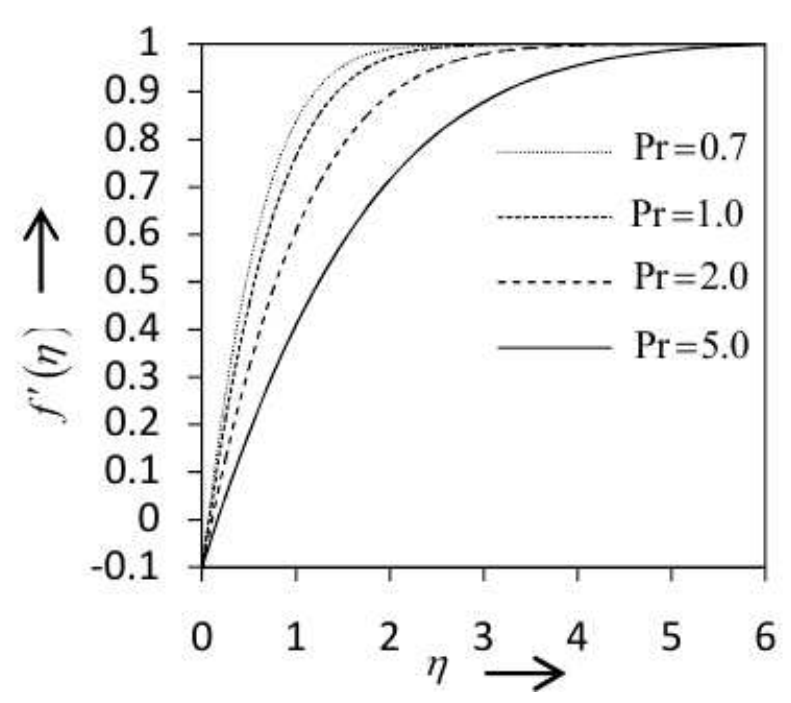

Figure 2: Variation of velocity profiles $f^{\prime}(\eta)$ against $\eta$ for several values of $\operatorname{Pr}$ with $K=0.1, M=0.1$ and $c=-0.1$ 


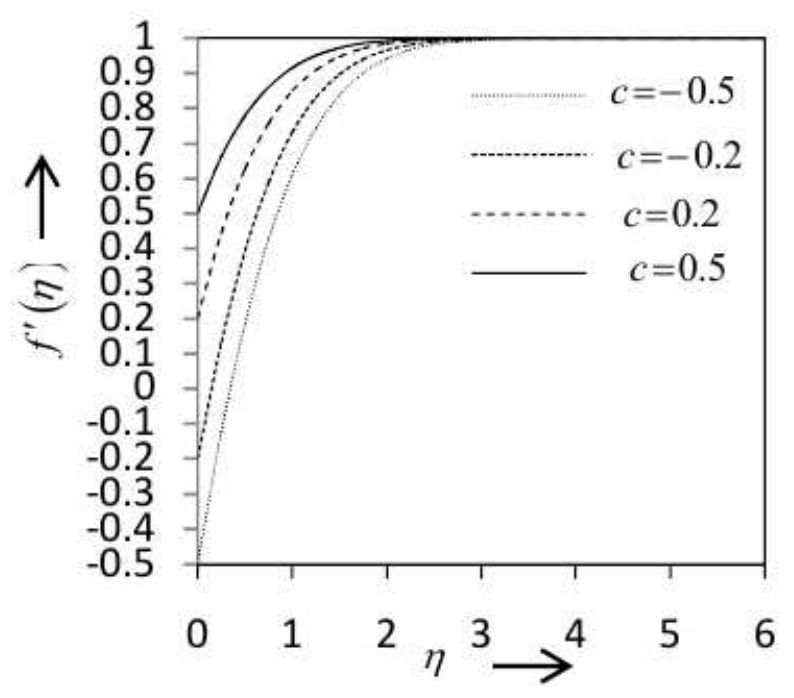

Figure 3: Variation of velocity profiles $f^{\prime}(\eta)$ against $\eta$ for several values of $K$ with $\operatorname{Pr}=1, M=0.1$ and $c=-0.1$

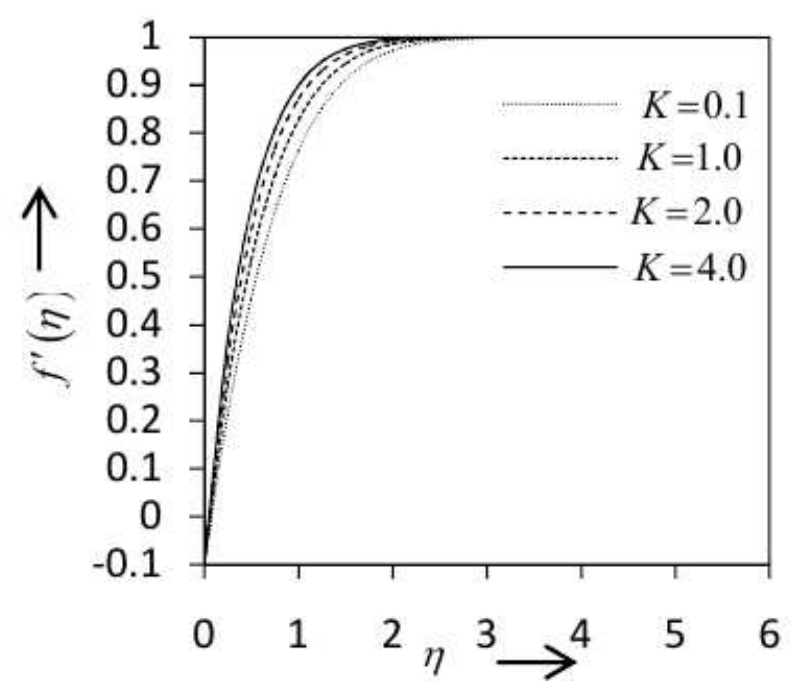

Figure 4: Variation of velocity profiles $f^{\prime}(\eta)$ against $\eta$ for several values of $M$ with $\operatorname{Pr}=1, K=0.1$ and $c=-0.1$ 


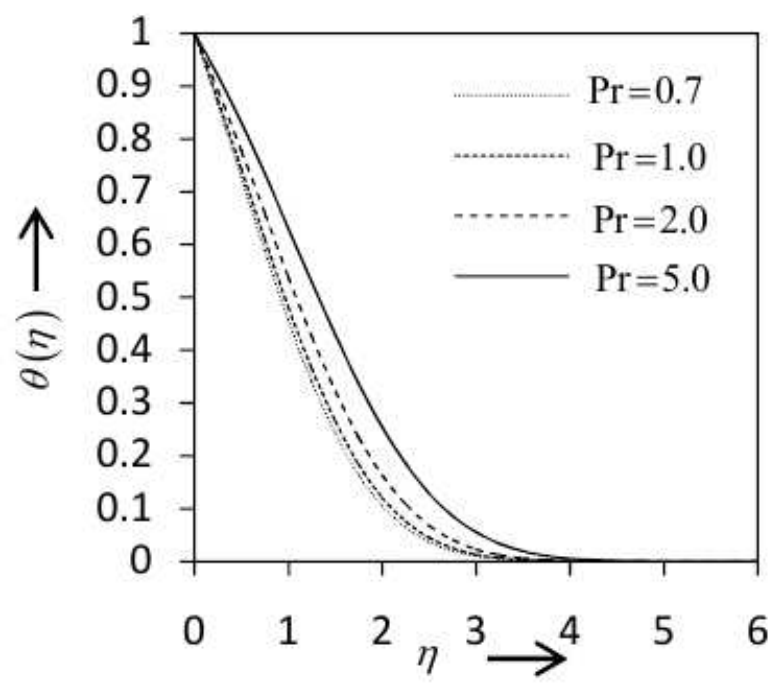

Figure 5: Variation of velocity profiles $f^{\prime}(\eta)$ against $\eta$ for several values of $c$ with $\operatorname{Pr}=1, K=0.1$ and $M=0.1$

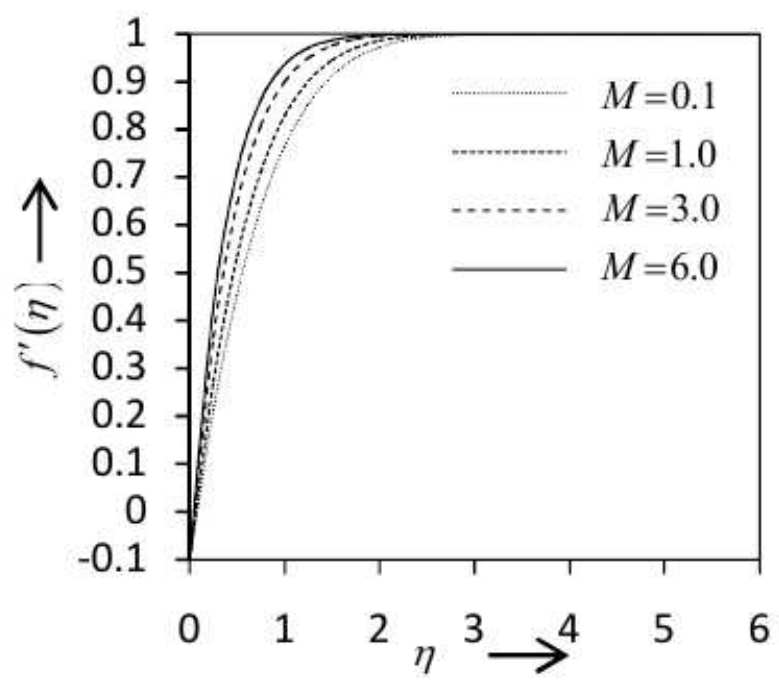

Figure 6: Variation of temperature profiles $\theta(\eta)$ against $\eta$ for several values of $\operatorname{Pr}$ with $K=0.1, M=0.1$ and $c=-0.1$ 


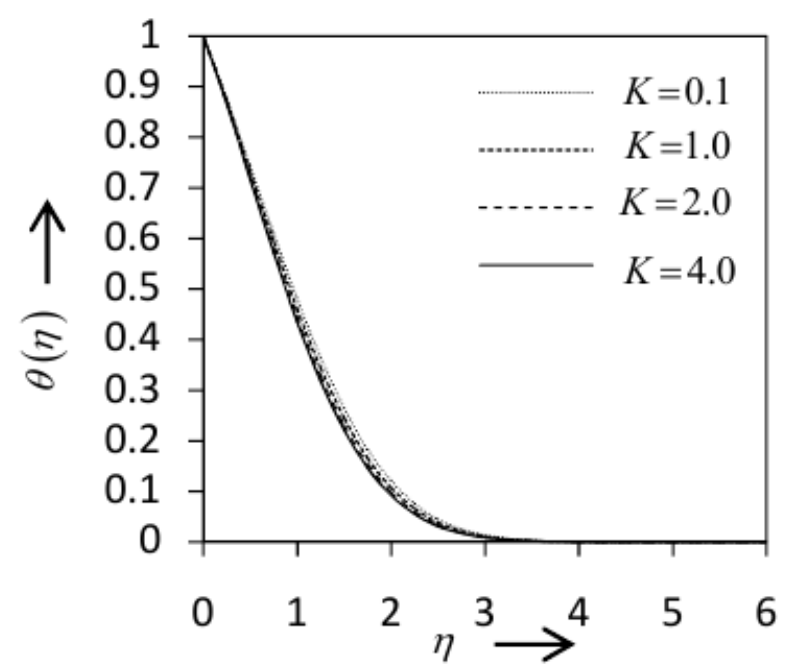

Figure 7: Variation of temperature profiles $\theta(\eta)$ against $\eta$ for several values of $K$ with $\operatorname{Pr}=1, M=0.1$ and $c=-0.1$

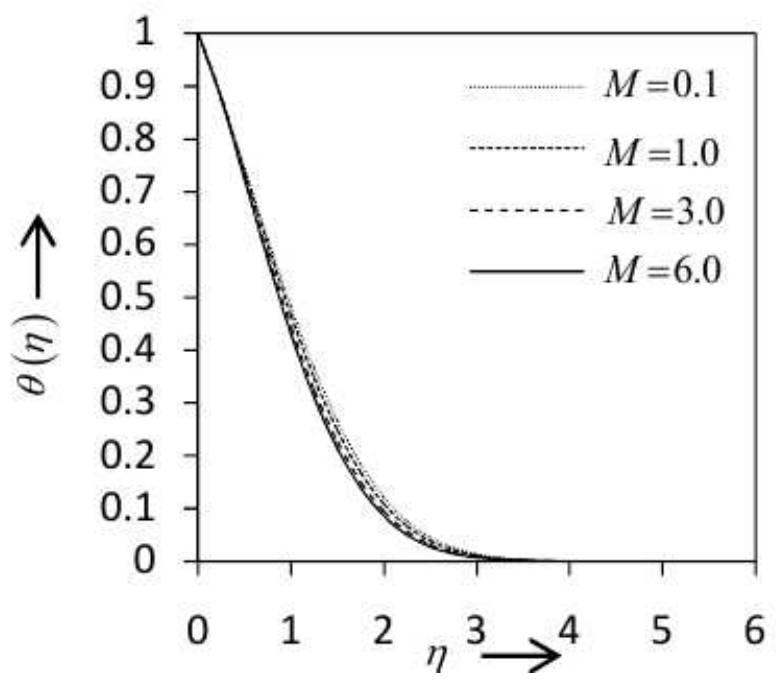

Figure 8: Variation of temperature profiles $\theta(\eta)$ against $\eta$ for several values of $M$ with $\operatorname{Pr}=1, K=0.1$ and $c=-0.1$ 


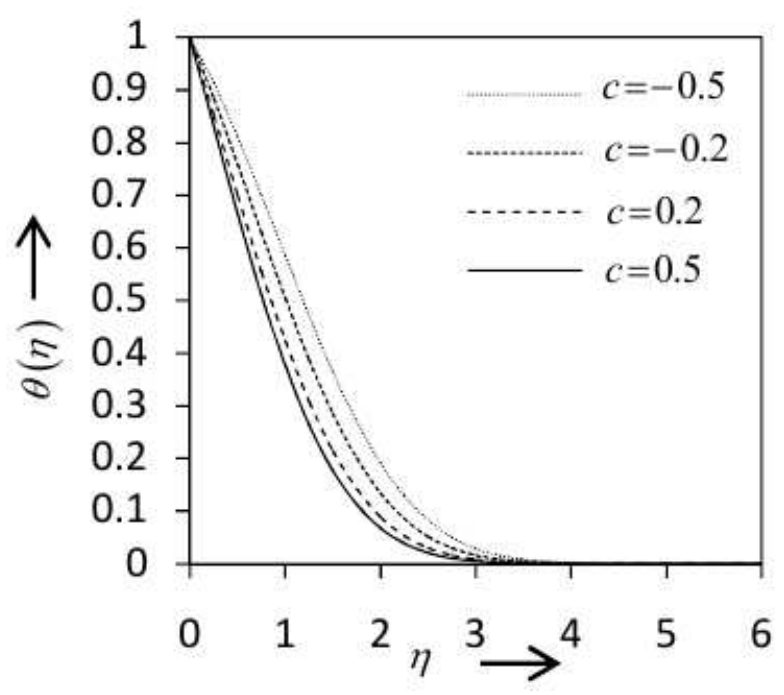

Figure 9: Variation of temperature profiles $\theta(\eta)$ against $\eta$ for several values of $c$ with $\operatorname{Pr}=1, K=0.1$ and $M=0.1$

\begin{tabular}{|c|c|c|c|c|c|}
\hline $\operatorname{Pr}$ & $K$ & $M$ & $c$ & $f^{\prime \prime}(0)$ & $-\theta^{\prime}(0)$ \\
\hline 0.7 & 0.1 & 0.1 & -0.1 & 1.67031 & 0.51104 \\
\hline 1 & & & & 1.39749 & 0.47611 \\
\hline 2 & & & & 0.98815 & 0.40193 \\
\hline 5 & & & & 0.62526 & 0.29199 \\
\hline 1 & 1 & 0.1 & -0.1 & 1.74282 & 0.48432 \\
\hline & 2 & & & 2.06021 & 0.48735 \\
\hline & 4 & & & 2.42609 & 0.48738 \\
\hline 1 & 0.1 & 1 & -0.1 & 1.74282 & 0.45734 \\
\hline & & 3 & & 2.33510 & 0.41844 \\
\hline & & 6 & & 2.98427 & 0.36429 \\
\hline 1 & 0.1 & 0.1 & -0.5 & 1.64007 & 0.31440 \\
\hline & & & -0.2 & 1.47457 & 0.43759 \\
\hline & & & 0.2 & 1.10973 & 0.58325 \\
\hline & & & 0.5 & 0.74709 & 0.67664 \\
\hline
\end{tabular}

Table 1: Numerical values of $f^{\prime \prime}(0)$ and $-\theta^{\prime}(0)$ for various values of $\operatorname{Pr}$, $K, M$ and $c$ 


\section{References}

[1] L.J. Crane, Flow past a stretching plate, Zeitschrift für Angewandte Mathematik und Physik, 21, No. 4 (1970), 645-647.

[2] P.S. Gupta, A.S. Gupta, Heat and mass transfer on a stretching sheet with suction or blowing, The Canadian Journal of Chemical Engineering, 55, No. 6 (1977), 744-746.

[3] H.I. Andersson, K.H. Bech, Magnetohydrodynamic flow of a power-law fluid over a stretching sheet, International Journal of Non-Linear Mechanics, 27, No. 6 (1992), 929936.

[4] E.M.A. Elbashbeshy, M.A.A. Bazid, Heat transfer over a stretching surface with internal heat generation, Canadian Journal of Physics, 81, No. 4 (2003), 699-703.

[5] R. Cortell, A note on magnetohydrodynamic flow of a power-law fluid over a stretching sheet, Applied Mathematics and Computation, 168, No. 1 (2005), 557-566.

[6] A. Ishak, R. Nazar, I. Pop, Hydromagnetic flow and heat transfer adjacent to a stretching vertical sheet, Heat and Mass Transfer, 44, No. 8 (2008), 921-927.

[7] R.N. Jat, S. Chaudhary, MHD flow and heat transfer over a stretching sheet, Applied Mathematical Science, 3, No. 26 (2009), 1285-1294.

[8] W. Ehlers, J. Bluhm, Porous Media: Theory, experiments and numerical applications, Springer, New York (2013).

[9] D.A. Nield, A. Bejan, Convection in porous media, $4^{\text {th }}$ edition, Springer, New York (2013).

[10] K. Vafai, C.L. Tien, Boundary and inertia effects on flow and heat transfer in porous media, International Journal of Heat and Mass Transfer, 24, No. 2 (1981), 195-203.

[11] T.C. Chiam, Stagnation-point flow towards a stretching plate, Journal of the Physical Society of Japan, 63, No. 6 (1994), 2443-2444.

[12] T.R. Mahapatra, A.S. Gupta, Magnetohydrodynamic stagnation-point flow towards a stretching sheet, Acta Mechanica, 152, No. 1-4 (2001), 191-196.

[13] T.R. Mahapatra, A.S. Gupta, Heat transfer in stagnation-point flow towards a stretching sheet, Heat and Mass Transfer, 38, No. 6 (2002), 517-521.

[14] A. Ishak, R. Nazar, I. Pop, Mixed convection boundary layers in the stagnation-point flow toward a stretching vertical sheet, Meccanica, 41, No. 5 (2006), 509-518.

[15] R.N. Jat, S. Chaudhary, Magnetohydrodynamic boundary layer flow near the stagnation point of a stretching sheet, Il Nuovo Cimento della Societa Italiana di Fisica, B: General Physics, 123, No. 5 (2008), 555-566.

[16] D. Pal, Heat and mass transfer in stagnation-point flow towards a stretching surface in the presence of buoyancy force and thermal radiation, Meccanica, 44, No. 2 (2009), $145-158$.

[17] C.Y. Wang, Stagnation flow towards a shrinking sheet, International Journal of NonLinear Mechanics, 43, No. 5 (2008), 377-382.

[18] T. Fang, J. Zhang, Thermal boundary layers over a shrinking sheet: an analytical solution, Acta Mechanica, 209, No. 3-4 (2010), 325-343.

[19] S. Chaudhary, P. Kumar, MHD slip flow past a shrinking sheet, Applied Mathematics, 4, No. 3 (2013), 574-581. 
[20] N.S. Akbar, Z.H. Khan, R.U. Haq, S. Nadeem, Dual solutions in MHD stagnation-point flow of Prandtl fluid impinging on shrinking sheet, Applied Mathematics and Mechanics, 35, No. 7 (2014), 813-820.

[21] K. Vafai, Handbook of porous media, $2^{\text {nd }}$ edition, Taylor \& Francis, US (2005).

[22] J.M.P.Q. Delgado, Heat and mass transfer in porous media, Springer, New York (2013).

[23] S.D. Harris, D.B. Ingham, I. Pop, Mixed convection boundary-layer flow near the stagnation point on a vertical surface in a porous medium: Brinkman model with slip, Transport in Porous Media, 77, No. 2 (2009), 267-285.

[24] D. Pal, P.S. Hiremath, Computational modeling of heat transfer over an unsteady stretching surface embedded in a porous medium, Meccanica, 45, No. 3 (2010), 415-424.

[25] H. Rosali, A. Ishak, I. Pop, Stagnation point flow and heat transfer over a stretching / shrinking sheet in a porous medium, International Communications in Heat and Mass Transfer, 38, No. 8 (2011), 1019-1032.

[26] T.R. Mahapatra, S.K. Nandy, Stability of dual solutions in stagnation-point flow and heat transfer over a porous shrinking sheet with thermal radiation, Meccanica, 48, No. 1 (2013), 23-32.

[27] S. Chaudhary, P. Kumar, MHD forced convection boundary layer flow with a flat plate and porous substrate, Meccanica, 49, No. 1 (2014), 69-77.

[28] S. Chaudhary, P. Kumar, Magnetohydrodynamic stagnation point flow past a porous stretching surface with heat generation, Indian Journal of Pure \& Applied Physics, 53, No. 5, (2015), 291-297.

[29] R.E. White, V.R. Subramanian, Computational methods in chemical engineering with maple, Springer-Verlag Berlin Heidelberg, Chennai, India (2010). 
